\title{
Electromagnetic resonance absorption in metallic gratings
}

\author{
V.M. Fitio ${ }^{1}$, H.P. Laba ${ }^{1}$ and Y.V. Bobitski ${ }^{1,2}$ \\ ${ }^{1}$ Lviv Polytechnic National University, 12, Bandera str., 79013 Lviv, Ukraine \\ Phone: +380322 582581, e-mail: polyana@polynet.lviv.ua \\ ${ }^{2}$ Institute of Technics, Rzeszow University, 16B, Tadeusza Rejtana str., 35-959 Rzeszow, Poland \\ Phone: +380322 582581, e-mail: bobitski@polynet.lviv.ua
}

\begin{abstract}
Reflection of electromagnetic waves with the $1.5 \mu \mathrm{m}$ length from a metallic grating (silver) with rectangular groove profile was analyzed using the method of coupled waves. Appearance of the waveguide effect in a dielectric film and, accordingly, of electromagnetic field resonance is a necessary condition of resonance absorption at presence of dielectric thin film on the grating. The electromagnetic field resonance phenomenon confirms a dependence of reflection on the wavelength, which is described by the Lorenz function. When the film is absent, strong absorption is possible at appearance of the waveguide effect between both grating metallic vertical walls. Due to plasma resonance, both the waveguide effect and, accordingly, high absorption arise at any distance between metallic walls of grating (slot width) for TM polarized waves. For this polarization in the slot of $0.478 \mu \mathrm{m}$ width filled with dielectric with permittivity $\varepsilon=9$, the two waveguide modes propagate. That fact is confirmed by a dependence of reflection logarithm on the grating depth. For TE polarized waves in the slot of $0.378 \mu \mathrm{m}$ width, the waveguide effect is possible on one mode only. The spectral dependence of reflection for TE polarized waves is well described by the Lorenz function, and due to excitation of two waveguide modes some deviation from this function is observed for TM polarized waves. The dependence of the reflection index on the grating depth completely agrees with the constant of waveguide mode propagation in the grating slot.
\end{abstract}

Keywords: resonance absorption, diffraction grating, coupled waves method, waveguide effect.

Manuscript received 09.03.06; accepted for publication 23.10.06.

\section{Introduction}

Recently due to interesting properties, different periodic structures including metallic films are widely investigated. The whole thematic sections in journals are devoted to this problem, for example the Optics Express 12, N16 (2004). Especially, the binary metallic gratings with narrow slots of width approximately ten times less than the grating period are studied widely [1-4]. The period of structures is commensurate with the wavelength. Transmission in those gratings is considerably higher than that can be foreseen, basing on the aperture theory [3,5]. These structures can be applied as optical filters, in submicron photolithography, in near-field microscopy [3], or biosensors [6]. Reasons for appearance or, in other words, the physical mechanism of anomalously high transmission in such a grating is discussed widely in scientific literature, but no final solution of this problem exists now [2, 4, 7-9].
Enough closed problems arise when analyzing reflection by metallic gratings with rectangular relief $[10,11]$. In the work [10], the results of theoretical researches of reflection by a metallic grating within the spectral range $0.21-3.2 \mu \mathrm{m}$ for metals that have a high reflection index. The numerical results were obtained by an approximate theory. It is shown that as a result of reflection of such a grating at certain wavelengths a field resonance is observed, which leads to a narrow dip in spectral characteristics of reflection. In this work, the researches are carried out only for TM polarized waves, and physical causes of resonance absorption appearance are not revealed, as well as a part of waveguides effects are not shown. The performed investigations of the spectral dependence for the reflection index of the metallic grating with a rectangular relief for several slot depths in [11]. In this work, the number analysis was conducted only for waves of TM polarization by approximate method. A comparison of experimental data 
with those obtained by the numerical method showed only qualitative coincidence of spectral characteristics. Also in this work, a part of waveguide effects at resonance absorption by the metallic grating is considered. In [12] reflection of a dielectric grating placed on the metallic substrate was studied. It was shown that at certain grating parameters, which characterizes the metallic substrate at the certain wavelength, the considerable absorption of the electromagnetic wave energy can be achieved. It was established that the necessary condition of this effect appearance consists in waveguide effect appearance or resonance of coupled waves in dielectric grating. The resonance presence is confirmed by both increasing of electric and magnetic fields in the grating by the factors of 5 to 20 concerning the incident wave amplitude and by the spectral reflection dependence described using the Lorenz function. In the work [12], the approximate parameters of the periodic structure was determined at complete absorption from the condition of the waveguide effect appearance [13]. Therefore, granting the results described elsewhere [10-12], we can assume that in grating depicted in Fig. 1, at certain parameters the resonance absorption of the electromagnetic wave energy is possible. At addition the waveguides effects perform an important part in the dielectric layer with thickness $d_{1}$ or in the slot with the width $a$. Hence, the purpose of this work consists in discovery of the part of waveguide effects in resonance absorption for metallic grating with rectangular relief and study of such an absorption feature for both light polarizations. Show that such absorption is possible at $d_{1} \neq 0$ and $d_{1}=0$. In the next sections, we will consider the mechanisms in these two different cases.

Analysis of reflection was conducted by the method of coupled waves (CWM) [14, 15] using the stable numerical algorithm [16, 17]. Our investigation was conducted at the following parameters: the normally incident wavelength $\lambda=1.5 \mu \mathrm{m}$, the dielectric permittivity $\varepsilon_{21}=9$, the permittivity of metal (silver) $\varepsilon_{22}=-121+i 6.6$ [18], and the fill coefficient $F=0.5$. Other parameters $\left(d_{1}, d_{2}, \Lambda\right)$ were determined by CWM calculation, granting no less than 53 diffraction orders. In the second section, the investigation results of absorption for a periodic structure with $d_{1} \neq 0$ is described. In the third section, the structure with $d_{1}=0$

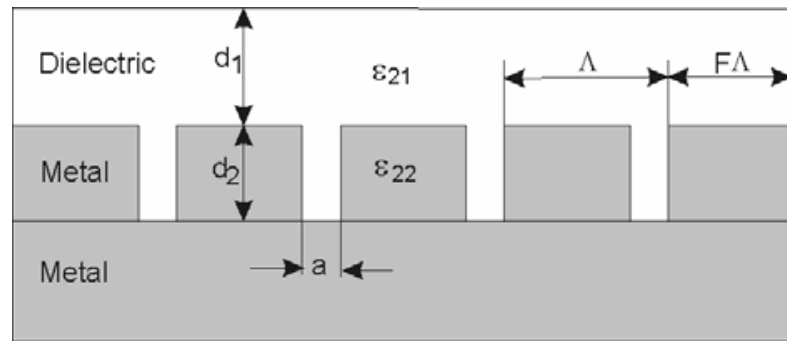

Fig. 1. Metallic grating with the rectangular relief. is considered and the structure investigation results are given. In the fourth section, the mechanisms of resonance absorption at $d_{1}=0$ is considered.

\section{Resonance absorption of electromagnetic waves in the dielectric film - metallic grating structure}

In the process of numerical calculations, it is necessary to find the values of parameters $d_{1}, d_{2}, \Lambda$, at which the electromagnetic wave complete absorption is observed in a grating. The initial values of these parameters were determined on conditions, that the waveguide effect appears [13], according to the rule [12]. According to this rule, the dependence $d_{1}=f(\Lambda)$ was built. Hence, the following initial approximations were used: $d_{1}=0.24 \mu \mathrm{m}$ and $\Lambda=0.671 \mu \mathrm{m}$ for $\mathrm{TE}$ polarized waves and $d_{1}=0.29 \mu \mathrm{m}$ and $\Lambda=0.95 \mu \mathrm{m}$ for $\mathrm{TM}$ polarized waves. Having $d_{1}$ and $\Lambda$ values, $d_{2}$ was determined by cut-and-try method with the purpose to obtain minimum reflection. Then $d_{1}$ and $\Lambda$ were changed again, and reflection of the grating was decreased, too. The parameters $d_{1}, d_{2}, \Lambda$ were found by these gradually approximations, when the reflection index was less than 0.001. Finally, the grating parameters $d_{2}$ and $\Lambda$ and dielectric layer thickness $d_{1}$ were determined using this way. These ones: $d_{1}=0.2027 \mu \mathrm{m}, \quad \Lambda=0.75575 \mu \mathrm{m}, \quad d_{2}=0.0254 \mu \mathrm{m}$ for $\mathrm{TE}$ polarization and $d_{1}=0.298 \mu \mathrm{m}$, $\Lambda=0.9559 \mu \mathrm{m}, d_{2}=0.0503 \mu \mathrm{m}$ for $\mathrm{TM}$ polarization. The reflection indexes for TE and TM polarizations were 0.000098 and 0.00041 , respectively.

Having the grating parameters of minimum reflection, calculated were the spectral reflection dependences for both polarizations, which is depicted in Fig. 2. The relative wavelength change is scaled on the abscissa axis.

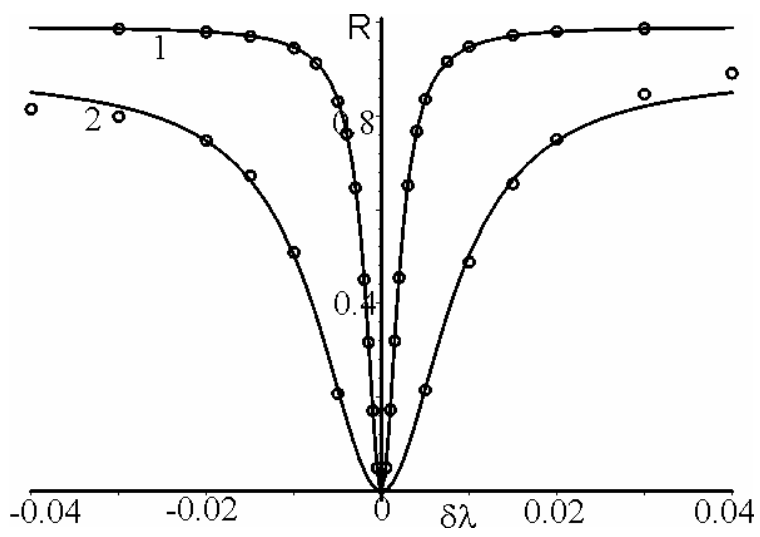

Fig. 2. The dependences of reflection from the grating on the wavelength relative change for TE (1), and TM (2) polarizations. 
In Fig. 2, the circles indicate the calculated points, and the continuous lines - approximation curves, which are described by following analytical expressions for TE and TM polarized waves, respectively:

$$
\begin{aligned}
& R(\delta \lambda)=0.99-\frac{0.99}{1+215000(\delta \lambda)^{2}}, \\
& R(\delta \lambda)=0.89-\frac{0.89}{1+130000(\delta \lambda)^{2}}
\end{aligned}
$$

where $\delta \lambda=\frac{1.5}{\lambda}-1$

As it follows from Fig. 2, the calculated points lie down very well on the fitted curve for TE polarized waves, and some deflections are observed for TM polarization. May be this difference is explained by the fact that $d_{2}$ is higher for TM polarization, and thus some perturbations in the behavior of the calculated dependence are caused. Such difference in the curves behavior induced to conduction of investigations of reflection on the grating depth $\left(d_{2}\right)$ at $d_{1}=0$. As a whole, from Fig. 2 we see that the spectral dependences are described by the Lorenz function, which testifies to the appearance of the waveguide effect in the dielectric layer. In other words, resonance of coupled waves arises in the waveguide.

\section{Electromagnetic waves absorption in rectangular relief metallic grating}

This section covers the investigation results of the dependence of reflection of metallic grating on the grating depth $d_{2}$, i.e. slots that are filled with dielectric possessing $\varepsilon_{21}=9$ at the wavelength of $1.5 \mu \mathrm{m}$. Other grating parameters correspond to the parameters from the section 2 . The dependences of reflection $(R)$ on $d_{2}$ for TE polarized waves are shown in Fig. 3a. The dependence of $\ln (R)$ on $d_{2}$ is represented in Fig. 3b, where the local minima from Fig. 3 a are denoted by circles. We see that these minima lie down very well on the straight line described by the following analytical expression:

$\ln (R)=-0.17-0.29 d_{2}$.
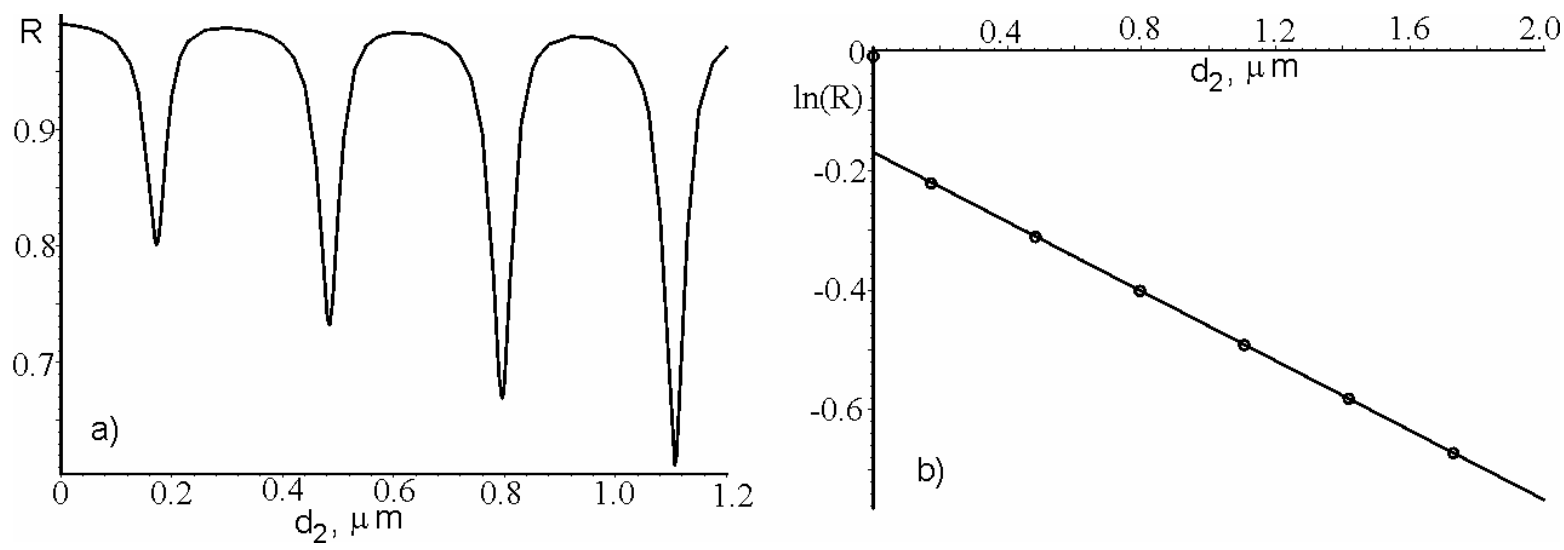

Fig. 3. The dependence of the reflection index on the grating depth for TE polarized waves.
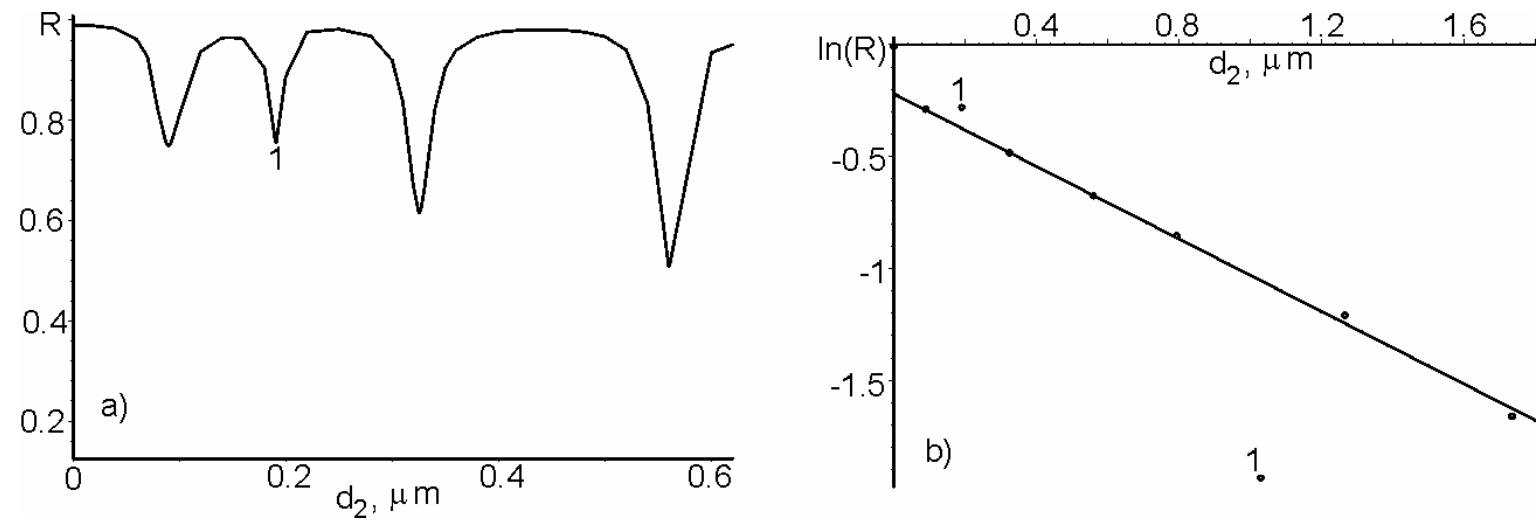

Fig. 4. The dependence of the reflection index on the grating depth for TM polarized waves. 
The first minimum is achieved at the depth $d_{2}^{(1)}=0.1726 \mu \mathrm{m}$. All other minima are achieved at the depths obtained from the following expression:

$d_{2}^{(n)}=d_{2}^{(1)}+(n-1) \Delta d_{2}$,

where $n$ - integer, and equal to unity or more than two, $\Delta d_{2}=0.3114 \mu \mathrm{m}$.

The analogous dependences for TM polarized waves are represented in Fig. 4. The straight line fitted to the circles (Fig. 4b) is analytically described as:

$\ln (R)=-0.22-0.81 d_{2}$.

From Fig. 4b, we see that the part of points lies down well on the straight line, and the points marked by the number 1 are aside it. In this case $d_{2}^{(1)}=0.0898 \mu \mathrm{m}$ and $\Delta d_{2}=0.2351 \mu \mathrm{m}$. The abscissas points aside the straight line are equal to: 0.191 and $1.03 \mu \mathrm{m}$. The corresponding difference of abscissas between these points is $0.839 \mu \mathrm{m}$.

The spectral dependences of the reflection index for two polarizations are shown in Fig. 5. For TE polarization (Fig. 5a) the grating depth is equal to $8.264 \mu \mathrm{m}$ that corresponds to $n=27$ according to the expression (3). The grating depth for TM polarization (Fig. 5b) is equal to $1.03 \mu \mathrm{m}$.

The calculated points denoted by circles are fitted by curves that for TE and TM polarizations are described by the following analytical expressions, respectively:

$$
\begin{aligned}
& R(\delta \lambda)=0.945-\frac{0.8943}{1+160000(\delta \lambda)^{2}}, \\
& R(\delta \lambda)=0.98-\frac{0.835}{1+7000(\delta \lambda)^{2}} .
\end{aligned}
$$

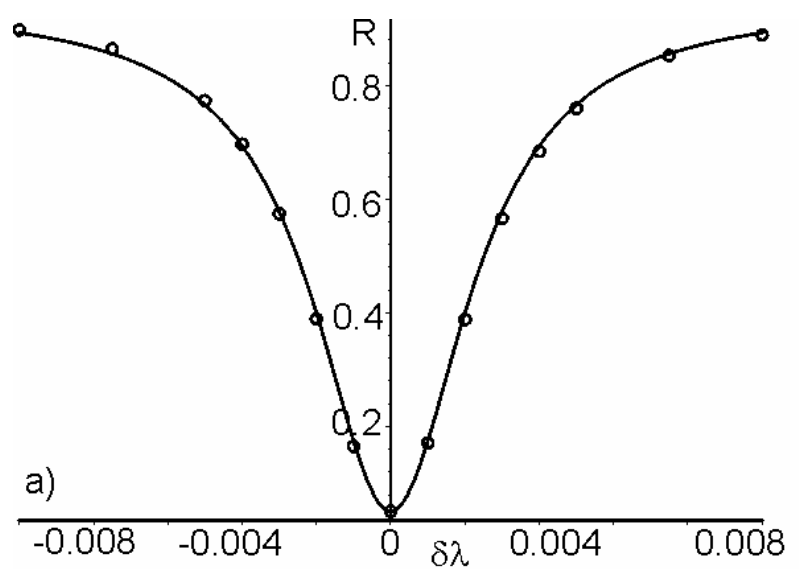

Again we see a good coincidence of the approximation curve for TE polarization and a worse coincidence in the case of TM polarization, especially, at little deviations from the wavelength $1.5 \mu \mathrm{m}$. But these curves give the possibility to express the opinion that the resonance phenomena become apparent as a result of maximum absorption. Also from Figs 3-5, we can conclude that for TE polarization one mode propagates, and for TM polarization two modes propagate in the slot. Modes have different propagation constants and different extinguishes. This statement will be proved in the next section.

\section{The resonance-waveguide phenomena in the grating slot}

The slot between two grating walls filled with dielectric, can be considered as a waveguide that possesses different characteristics of waveguide modes propagation for different polarizations. The data characterizing this difference are shown in Fig. 6 (Figs 6a, b, c are concerned to TM polarization, Fig. 6d corresponds to TE polarization). In Fig. 6a, the dependence of wave reflection of the interface of dielectric-metal on grazing angle (sum of grazing and incidence angles is equal to $\pi / 2$ ) is depicted. At the angle $0.35 \mathrm{rad}$, the reflection minimum is observed. Both the dependences of the reflected beam phase for the interface dielectric-metal (curve 1) and the dependence of the slot width $a$ (curve 2), at which the waveguide effect can be possible, on the grazing angle are represented in Figs 6b-d. The horizontal line 3 corresponds to slots with 0.478 and $0.378 \mu \mathrm{m}$ widths for TM and TE polarizations, respectively, at which the calculation of the reflection dependence on the slot width was performed. The phase and the slot width calculations were carried in accordance with expressions

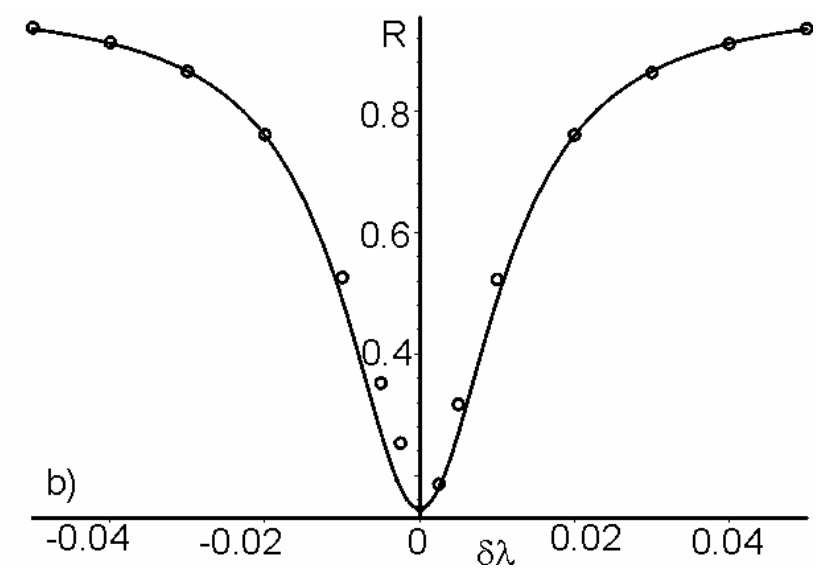

Fig. 5. The dependences of the reflection indexes on the wavelength relative change for TE (a) and TM (b) polarizations. 
from [13], In the case of the slot width $a$, the corresponding expression has the following form:

$a=\frac{\lambda(\Phi(\alpha)+\pi m)}{\pi \sqrt{\varepsilon_{21}} \sin \alpha}$,

where $\alpha$ is the angle of grazing, $\Phi$ is the reflected beam phase for the interface dielectric-metal, $m$ is the integer, for example, $0,1, \ldots$

Hence, for TM polarization, in the slot of $0.478 \mu \mathrm{m}$ width only two modes can propagate at $m=1$ (Fig. 6b) and $m=2$ (Fig. 6c). At other $m$, the slot width $0.478 \mu \mathrm{m}$ does not cross the curve $a(\alpha)$ when changing $\alpha$ from 0 to $\pi / 2$. It should be paid attention to Fig $6 b$. At the grazing angle $0.35 \mathrm{rad}$, both the phase jump $\Phi$ within $2 \pi$ and $0.378 \mu \mathrm{m}$ corresponding jump in the dependence of the slot width on the grazing angle are observed. Comparing Figs $6 \mathrm{a}$ and $6 \mathrm{~b}$, we can conclude that behavior of curves is typical for resonance phenomena, i.e., in this case we have the so-called plasmon resonance. Incidentally note, if $m=0$, the jump of $a$ is observed within the limits from 0 to
$0.36 \mu \mathrm{m}$, i.e., for TM polarization the waveguide effect between metallic walls at an arbitrary slot width can be possible. The latter fact explains an anomalously high transmission of the metallic grating with narrow slots [2, $3,5]$. At $m=1$ and at the slot width $0.378 \mu \mathrm{m}$, the only waveguide mode is possible for TE polarized waves, which is demonstrated in Fig. 6d.

To find the modes propagation constants $\beta$, we used the expressions from [13] for TE and TM polarizations, respectively:

$D(\beta)=w(u \cos u+v \sin v)-u(\sin ) u-v \cos u$,

$D(\beta)=\frac{w}{\varepsilon_{21}}\left(\frac{u}{\varepsilon_{22}} \cos u+\frac{v}{e_{22}} \sin u\right)-$

$-\frac{u}{\varepsilon_{22}}\left(\frac{u}{\varepsilon_{22}} \sin u-\frac{v}{\varepsilon_{22}} \cos u\right)$,

where $\quad u=a \sqrt{\varepsilon_{21} k^{2}-\beta^{2}}, \quad w=v=a \sqrt{\varepsilon_{22} k^{2}-\beta^{2}}$, $k=2 \pi / \lambda, i=\sqrt{-1}$

According to [13], modes can propagate in the waveguide only with the propagation constant $\beta$, if
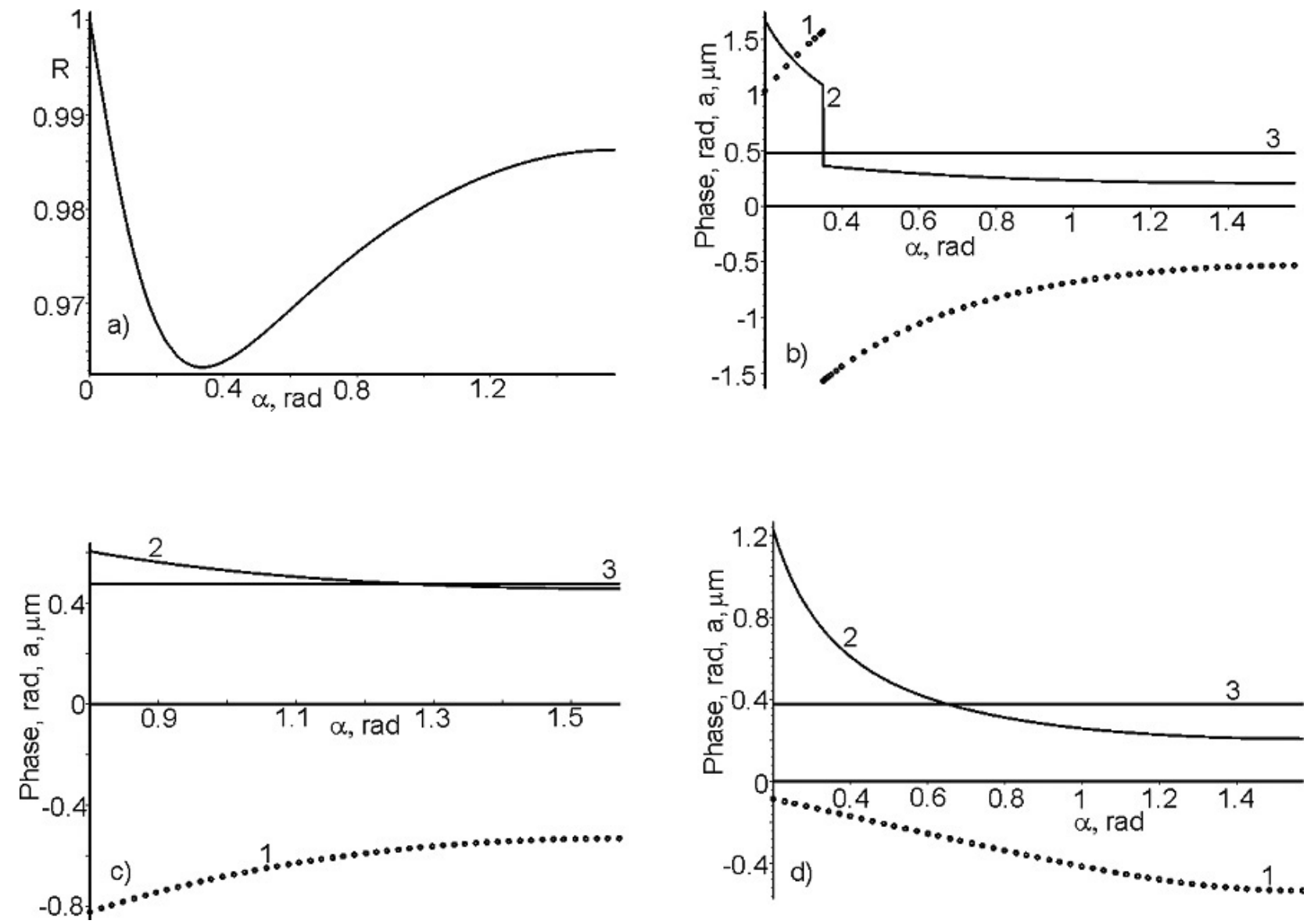

Fig. 6. The dependences of ray phase reflected from the interface dielectric-metal (curve 1) and the slot width (curve 2) on the grazing angle; straight lines 3 correspond to the slot widths. 


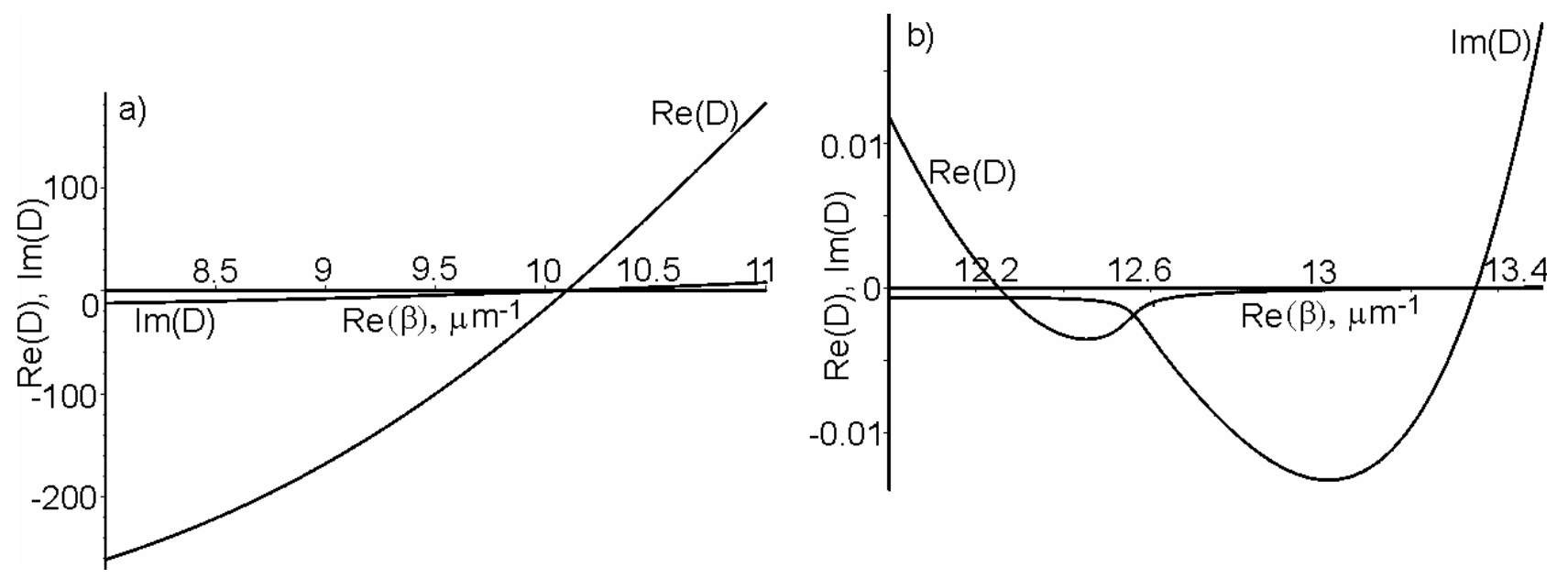

Fig. 7. The dependences of $\operatorname{Re}(D)$ and $\operatorname{Im}(D)$ on $\operatorname{Re}(\beta)$ for $\mathrm{TE}$ (a) nad TM (b) polarizations.

$D(\beta)=0$. Owing to absorption, the propagation constant can be represented as: $\beta=\operatorname{Re} \beta+i \operatorname{Im} \beta$, in this case $\gamma=\operatorname{Im} \beta$ is the extinction index. In Fig. 7 (Fig. 7a TE polarization, Fig. $7 \mathrm{~b}-\mathrm{TM}$ polarization) the dependences of $D(\operatorname{Re} \beta)$ on $\operatorname{Re}(\beta)$ are shown for $\gamma$ at which the $\operatorname{Re}(\beta)$ will be found, when $D(\beta)=0$. The wave of TE polarization that propagates into the grating slot has the following propagation constant:

$\beta=10.096-i 0.01143$.

For TM polarization, we have two propagation constants according to Figs $4 \mathrm{~b}$ and 6 :

$\beta_{1}=13.348-i 0.0248, \beta_{2}=3.804-i 0.1062$,

where $\beta_{2}$ corresponds to $m=2$ according to the expression (6). This waveguide mode disappears at decreasing of the slot width down to $0.45 \mu \mathrm{m}$, and one mode is kept, which corresponds to $m=1$ and can be caused by excitation of plasmons.

The local minimum in the dependence of the reflection index on the grating depth corresponds to that being formed in the slot standing wave. The wave period can be determined using the expression:

$\Delta d_{2}=\frac{\pi}{\operatorname{Re}(\beta)}$.

According to expressions (10) and (11) $\Delta d_{2}$ can be defined, which for TE polarization is equal to $0.3112 \mu \mathrm{m}$ and coincides with the value $0.3114 \mu \mathrm{m}$ very well as calculated using table data. Being based on the latter data, Fig. 3 was built. The values of $\Delta d_{2}$ for TM polarization calculated using the expressions (10) and (11) are equal to 0.2354 and $0.826 \mu \mathrm{m}$; corresponding $\Delta d_{2}$, which were obtained using the table data, have the following magnitudes: 0.2351 and $0.839 \mu \mathrm{m}$. Again we see the good coincidence of $\Delta d_{2}$ obtained by both methods for $0.2354 \mu \mathrm{m}$ (difference exists only in the fourth sign) and the worse coincidence for $0.826 \mu \mathrm{m}$ (difference in the second sign). That can be explained by the fact that the grating depth $1.03 \mu \mathrm{m}$ for both modes is closed to maximum excitation. For example: $d_{2}^{(4)}=d_{2}^{(1)}+(n-1) \Delta d_{2}=0.0898+4 \times 0.2354$ $=1.031 \mu \mathrm{m}$ and $d_{2}^{(2)}=d_{2}^{(1)}+(n-1) \Delta d_{2}=0.191+$ $1 \times 0.826 \mu \mathrm{m}=1.017 \mu \mathrm{m}$.

From the latter calculations, we really see that both modes are excited simultaneously, though they are not in their own "optimum" regimes, therefore there is some difference when determining $\Delta d_{2}$ for the mode that corresponds to $m=2$.

Hence, the presence of the both modes also explains the feature of the spectral dependence for the reflection index for TM polarization (Fig. 5b). One of the modes approximates to "optimum" regime at negligible deviation of the wavelength from $1.5 \mu \mathrm{m}$, and other ones deflect from it. It is needed to account that these modes have the different losses at propagation. All together lead to asymmetry of spectral characteristics and their deviation from the Lorenz function. For TE polarization, where only one mode propagates, the spectral characteristics agrees to the Lorenz function at reflection of the grating very well (see Fig. 5a).

\section{Conclusions}

Both waveguide effect appearance in the dielectric film and, accordingly, electromagnetic field resonance appearance are the necessary condition of resonance absorption at presence of thin dielectric film on the metallic grating. The phenomenon of electromagnetic field resonance confirms the dependence of reflection on the wavelength, which is described by the Lorenz function. At addition, for example, for TE polarization, the film width $\left(d_{1}=0.298 \mu \mathrm{m}\right)$ and the grating depth 
$\left(d_{2}=0.0254 \mu \mathrm{m}\right)$ at complete absorption is lower than the wavelength. At film absence, strong absorption is achieved at waveguide effect appearance in the slot that acts as a microresonator. In this microresonator, the standing wave is formed, and the resonance slot depth is determined by the expression $d_{2}^{(n)}=d_{2}^{(1)}+(n-1) \Delta d_{2}$. The standing wave period $\Delta d_{2}$ is determined by the real part of the propagation constant $\beta$ as $\Delta d_{2}=\frac{\pi}{\operatorname{Re}(\beta)}$. For TM polarized waves, the waveguide effect and, accordingly, high absorption appear at any slot width due to plasmon resonance. There is the minimum grating width, at which the waveguide effect is possible and, accordingly, high absorption for TE polarization. Those grating structures from metals with low imaginary part of the dielectric constant (for example, $\mathrm{Au}, \mathrm{Ag}, \mathrm{Cu}, \mathrm{Na}$, $\mathrm{K})$ can serve as a basis for creation of different sensors, including biosensors. Point is that slight changes of grating structure parameters or the refraction index of medium, in which the incident wave propagates, strongly influence on reflection of this periodic structure.

\section{References}

1. J.M. Steele, C.E. Moran, A. Lee, C.M. Aguirre and N.J. Halas, Metallodielectric gratings with subwavelength slots: Optical properties // Phys. Rev. B 68, p. 205103-205109 (2003).

2. E. Popov, M. Nevière, S. Enoch and R. Reinisch, Theory of light transmission through subwavelength periodic hole array // Phys. Rev. B 62(23), p. 16100-16108 (2000).

3. J. A. Porto, F.J. Garcia-Vidal and J.B. Pendry, Transmission resonances on metallic gratings with very narrow slits // Phys. Rev. Lett. 83(14), p. 2845 2848 (1999).

4. N. Bonod, S. Enoch, L. Li, E. Popov and M. Naviere, Resonant optical transmission through thin metallic films with and without holes // Optics Express 11(5), p. $482-490$ (2003).

5. L. Martin-Moreno, F.J. Garcia-Vidal, H.J. Lezes, K.M. Pellerin, T. Thio, J.B. Pendry and T.W. Ebbesen, Theory of extraordinary optical transmission through subwavelength hole arrays // Phys. Rev. Lett. 86(6), p. 1114-1117 (2001).

6. K.M. Byun, S.J. Kim and D. Kim, Design study of highly sensitive nanowire enhanced surface plasmon resonance biosensors using rigorous coupled wave analysis // Optics Express 13(10), p. 3737-3743 (2005).

7. M.M.J. Treacy, Dynamical diffraction explanation of the anomalous transmission of light through metallic gratings // Phys. Rev. B 66, p. 195105 195115 (2002).

8. D. Gerard, L. Salomon, F. De Fornel and A.V. Zayats, Analysis of the Bloch mode spectra of surface polaritonic crystals in the weak and strong coupling regimes: grating-enhanced and suppression of SPP radiative losses // Optics Express 12(16), p. 3652-3663 (2004).

9. Q. Cao and Ph. Lalane, Negative role of surface plasmons in the transmission of metallic grating with narrow slits // Phys. Rev. Lett. 88(5), p. 057403-057406 (2002).

10. D.C. Skigin, A.N. Fantino and S.I. Grosz, Phase resonance in compound metallic gratings // J. Opt. A: Pure and Appl. Opt. 5, p. S129-S135 (2003).

11. T. López-Rios, D. Mendoza, V.J. García-Vidal, J. Sánchez-Dehesa and B. Pannetier, Surface shape resonance in lamellar metallic gratings // Phys. Rev. Lett. 81(3), p. 665-668 (1998).

12. V.M. Fitio and Y.V. Bobitski, Resonance effects in a dielectric grating; total absorption of electromagnetic waves by a dielectric grating on metal system // J. Opt. A: Pure Appl. Opt. 6, p. 943-951 (2004).

13. H.-G. Unger, Planar optical waveguides and fibres. Mir, Moscow, 1980 (in Russian).

14. T.K. Gaylord, and M.G. Moharam, Analysis and applications of optical diffraction by grating // Proc. IEEE 73(5), p.894-937 (1985).

15. L. Li, Use of Fourier series in the analysis of discontinuous periodic structures // J. Opt. Soc. Amer. A 13(9), p. 1870-1876 (1996).

16. M.G. Moharam, E.B. Grann, D.A. Pommet and T.K. Gaylord, Formulation for stable and efficient implementation of the rigorous coupled-wave analysis of binary grating // J. Opt. Soc. Amer. A 12(5), p. 1068-1076 (1995).

17. V.M. Fitio, H.P. Laba and Y.V. Bobitski, The band structure analysis of $1 \mathrm{D}$ and $2 \mathrm{D}$ photonic crystals by coupled wave method // Radiophysics and Electronics 10(1), p. 123-131 (2005).

18. P.B. Jonson and R.W. Christy, Optical constants of the noble metals // Phys. Rev. B 6(12), p. 43704379 (1972) 